\title{
The Effectiveness of Frequency-Based Resistance Training Protocols on Muscular Performance and Hypertrophy in Trained Males: A Critically Appraised Topic
}

\author{
Theodore Kent Kessinger, Bridget Melton, Theresa Miyashita, and Greg Ryan
}

\begin{abstract}
Clinical Scenario: Manipulation of exercise variables in resistance training (RT) is an important component in the development of muscular strength, power, and hypertrophy. Currently, most research centers on untrained or recreationally trained subjects. This critically appraised topic focuses on studies that center on the well-trained subject with regard to frequency of training. Clinical Question: In well-trained male subjects, is there an association between RT frequency and the development of muscular strength and hypertrophy? Summary of Key Findings: Four studies met the inclusion criteria and were included for analysis. All studies showed that lower-frequency training could elicit muscular strength and hypertrophy increases. One study suggested that a higher frequency compared with a lower frequency may provide a slight benefit to hypertrophic development. One study reported a greater level of delayed onset muscle soreness with lower frequency training. The 4 studies demonstrate support for the clinical question. Clinical Bottom Line: Current evidence suggests that lower-frequency RT produces equal to greater improvements on muscular strength and hypertrophy in comparison to higher-frequency RT when volume is equated. The evidence is particularly convincing when lower-frequency RT is associated with a total-body training protocol in well-trained male subjects. Strength of Recommendation: There is moderate-to-strong evidence to suggest that lower-frequency RT, when volume is equated, will produce equal to greater improvements on muscular strength and hypertrophy in comparison to higherfrequency RT.
\end{abstract}

Keywords: total body, split routine, training frequency

\section{Clinical Scenario}

Resistance training (RT) is a common and well-accepted method for the development of muscular strength, power, and hypertrophy. Numerous studies have been undertaken to provide insight into the differences between the frequency of training, ${ }^{1-6}$ number of muscle groups exercised, ${ }^{7,8}$ training volume, ${ }^{9-14}$ rest intervals, ${ }^{15-17}$ and velocity of the weight being lifted, ${ }^{18}$ although many of these studies have focused on the untrained or novice recreational athlete. ${ }^{1,3,9,16}$ In the untrained or novice subject, RT can produce substantial gains in a short period of exposure to a training protocol. Studies have shown that a great deal of muscle growth occurs in the initial weeks of training, ${ }^{4,19-21}$ primarily in neural adaptations but also in muscle hypertrophy. ${ }^{21}$ As the muscle begins to adapt to RT, muscle growth attenuates, as it becomes difficult to provide a more effective stimulus to increase muscle growth. $4,22,23$ This is the paradigm that the well-trained subject must overcome to obtain significant training improvements.

The well-trained subject must follow RT protocols that manipulate training variables, such as training frequency, volume, and others, to promote additional improvements beyond muscle growth and adaptation plateaus. Manipulation of training frequency has been proposed as an effective stimuli to provide adaptations beyond the initial training., ${ }^{2,46,24}$ Training volume has also been suggested as an appropriate means of increasing muscular adaptations, such as

Kessinger is with the Ottawa University, Ottawa, KS, USA. Kessinger, Melton, and Miyashita are with the Concordia University Chicago, River Forest, IL, USA. Melton and Ryan are with the Georgia Southern University, Statesboro, GA, USA. Kessinger (kentkessinger50@gmail.com) is corresponding author. hypertrophy. ${ }^{10,12}$ What is unclear is whether manipulation of the training frequency or manipulation of the volume of exercises provides the most effective protocol for well-trained subjects.

In addition, much of the research that has been previously done has included split-routine (SR; or split-body) protocols. .,22,25 $^{2}$ Although limited in scope, there is some evidence purporting the implementation of total-body (TB) training protocols in conjunction with reduced training frequency per week in trained individuals, particularly males. ${ }^{6,26,27}$ Understanding the research into frequency and volume manipulation in comparison to SR and TB routines could have a profound effect on training recommendations for trained individuals, particularly those in male athletic sports, where the development of power through RT is at a premium. Therefore, the purpose of this critically appraised topic (CAT) was to review the recent evidence in training frequency (controlling for volume) on the development of performance indices, such as muscular strength and hypertrophy, in the welltrained male resistance-trained subjects.

\section{Focused Clinical Question}

In well-trained male subjects, is there an association between RT frequency and the development of muscular strength and hypertrophy?

\section{Summary of Search, "Best Evidence" Appraised, and Key Findings}

- The literature was searched for studies of level 2 evidence or higher (based on Oxford Center of Evidence-Based Medicine 
2011, Levels of Evidence ${ }^{28}$ ) that compared frequency as a training method for the development of muscular strength and hypertrophy.

- Six randomized controlled trials met the inclusion and exclusion criteria and then were further screened for methodology. 4,6,10,26,29,30 One article was excluded, as it did not control for training volume, ${ }^{30}$ and another, as it used a daily undulating periodization plan. ${ }^{10}$

- Four articles were chosen to be included in the CAT.4,6,26,29

\section{Clinical Bottom Line}

There is moderate-to-strong evidence to suggest that a lowerfrequency RT program, when volume is equated, will produce equal and, in some instances, greater improvements on muscular strength and hypertrophy in comparison to higher-frequency RT. The evidence is particularly convincing when lower-frequency RT is combined with a TB training protocol in well-trained male RT subjects.

\section{Strength of Recommendation}

Based on the Oxford Center for Evidence-Based Medicine strength of recommendation, ${ }^{29}$ there is level A evidence to support the use of lower-frequency RT protocols for the development of muscular strength and muscle hypertrophy in trained males. The results were consistent across all 4 studies included in this appraisal.

\section{Search Strategy}

\section{Terms Used to Guide Search Strategy}

- Patient/Client group: trained males OR resistance-trained males

- Intervention: TBRT OR split-body routine

- Comparison: training frequency (high OR low)

- Outcome: performance gains (muscular strength OR hypertrophy)

\section{Sources of Evidence Searched}

- EBSCOhost

- Academic Search Complete

- SPORTDiscus

- PubMed

- Health Source

- Google Scholar

- Additional articles obtained through review of reference lists

\section{Inclusion and Exclusion Criteria}

\section{Inclusion Criteria}

- Date range: 2014-2019 (time frame selected to include most recent evidence)

- Peer-reviewed or academic journal articles, full text
- Inclusion terms: resistance training, strength training, weight training, resistance exercise, frequency, trained men/males, TB (or full body or whole body), SR (or split body, or upper body/ lower body)

- English language

- Randomized controlled trials

\section{Exclusion Criteria}

- Chronic disease or physical injury studies

- Studies that centered on females, menopause, or untrained individuals

- Single exercise comparisons

- Unequal training volume

- Meta-analysis, systematic reviews, reviews of literature, or position statements

\section{Results of Search}

Four relevant studies 4,6,26,29 were identified and categorized in Table 1 (based on Levels of Evidence, Center for Evidence-Based Medicine). ${ }^{31}$

\section{Best Evidence}

The studies selected for inclusion in this CAT are listed in Table 2. The 4 studies included were identified as the best evidence and selected as the most appropriate, given the inclusion criteria and focused clinical question. The PEDro scale was used to assess the quality index of the studies. This scale has been validated and is used to assess the methodological quality of clinical trials. ${ }^{31,32}$

\section{Implications for Practice, Education, and Future Research}

Previous research has explored modifications to training volume and frequency, ${ }^{1-6,10-14}$ but very few have studied the effects of training manipulation on well-trained subjects. The articles reviewed in this CAT reported frequency differences while controlling for volume, the number of exercises, or the muscle groups trained.4,6,26,29 The results from this appraisal support both the lower- and higher-frequency RT protocols for the development of muscle hypertrophy and other performance measures, such as muscular strength. However, in 3 of the 4 studies, the groupings that were considered low frequency produced greater gains in both 1 repetition maximum bench press and 1 repetition maximum squat. 4,6,29 The most important implication for practice is that welltrained RT males can benefit from a lower training frequency per week (number of sessions per week), particularly if the volume is increased. ${ }^{29}$

As asserted in one of the focused research articles, about twothirds of muscle growth occurs in the first weeks of RT, and through adaptive processes by the muscles, the rate of growth reduces. ${ }^{4}$ Those RT subjects who are well trained may not provide the muscle with enough stimuli to increase muscle growth. However, it appears employing a training protocol with a low frequency per week that increases the total volume per training session may 
Table 1 Summary of Study Designs of Articles Retrieved

\begin{tabular}{|c|c|c|}
\hline Level of evidence $^{a}$ & Study design/methodology of articles retrieved & Study \\
\hline $1 b$ & Test-retest repeated measures and correlation design & Gomes et $\mathrm{al}^{4}$ \\
\hline $1 b$ & Test-retest repeated measures and correlation design & Schoenfeld et $\mathrm{al}^{6}$ \\
\hline $1 b$ & Test-retest repeated measures and correlation design & Brigatto et $\mathrm{al}^{26}$ \\
\hline $1 b$ & Test-retest repeated measures and correlation design & Yue et $\mathrm{al}^{29}$ \\
\hline
\end{tabular}

${ }^{\mathrm{a}}$ Level of evidence assessed using the Oxford Center for Evidence-based Medicine 2011 criteria. $^{28}$

produce body mass enhancements, as well as increases in strength, particularly upper-body strength. ${ }^{29}$ Yue et al ${ }^{29}$ suggest that higher metabolic stress associated with a high-volume low-frequency protocol may represent the stimulus necessary to create an enhanced anabolic response by skeletal muscle. Increased volume routines have been associated with a greater posttraining increase in growth hormone and testosterone, accentuating the potential for muscle tissue remodeling. ${ }^{29}$

The evidence from the CAT articles provides a strong rationale for lower-frequency training. It should also be noted that protocols that are structured with a lower weekly training frequency could be beneficial to the time-restricted athlete or RT subject. Often, time constraints, like those of the college athlete, do not allow for daily training that could elicit the needed physiological response to RT for substantial muscular growth or adaptations. Increased training volume in subsequent training sessions, with adequate recovery time between sessions, could be an appropriate consideration, considering that it would be "day saving" and induce hypertrophic, body composition, and muscular strength gains.

Another notable RT protocol manipulation from the CATfocused articles was that 2 of the 4 studies included SR versus TB routine protocols. The TB protocols were a component of the lower-frequency training protocols. The American College of Sports Medicine, in their position statement, recommends a frequency of $3 \mathrm{~d} / \mathrm{wk}$ if training is following a TB protocol, and a frequency of $4 \mathrm{~d} / \mathrm{wk}$ if training utilizes an SR protocol. ${ }^{22}$ In comparing the SR to the TB in the articles, it was found that they produced similar results, with the TB showing greater hypertrophic results in the Schoenfeld et $\mathrm{al}^{6}$ research study. The Gomes et $\mathrm{al}^{4}$ study produces similar increases between SR and TB routines, but higher levels of delayed onset muscle soreness were observed in the TB protocol as compared with the SR protocol. It was suggested that the higher daily volume present in the TB protocol may have influenced the increased delayed onset muscle soreness, whereas a repeated bout effect from the SR protocol may have contributed to a protective effect against delayed onset muscle soreness. ${ }^{4}$

The current study has some limitations that offer opportunities for future research. The studies in this CAT show that a lower frequency of training sessions will produce muscular strength and hypertrophy improvements; however, only 2 of the studies compared the SR protocol to the TB protocol. ${ }^{4,6} \mathrm{SR}$ protocols have been a staple in both powerlifting and weight training and have been a recommended protocol by the American College of Sports Medicine for RT in healthy adults. ${ }^{22}$ Recently, TB protocols have become a popular method of RT, particularly with trained athletes, ${ }^{33}$ and further comparisons should be undertaken. Also, the studies themselves ranged from a low of 9 exercises utilized ${ }^{26}$ to a high of 21 exercises utilized ${ }^{6}$ to produce outcome measures for strength improvement and hypertrophy changes. The wide range of exercises cause some limitations for direct comparison between each of the studies. Even though volume was constant within all studies, the volume between studies varied greatly due to the number of exercises prescribed. Finally, another limitation was that the training frequency ranged from a minimum of 2 sessions per week found in 2 studies 26,29 to 1 study, which had 5 training sessions per week for all study subjects. ${ }^{4}$ Meta-analytical data have shown that training a muscle group 2 times per week in well-trained individuals produced the highest effect size. ${ }^{22,34}$

Each of the studies used 1 repetition maximum of both the bench press and back squat as standards for muscular strength. The back squat is well recognized as a useful exercise in assessing lower-body muscular strength, as it involves the movement of several joints (ankle, knee, and hip joints), as well as engaging a large number of lower-body muscle groups. ${ }^{25,35}$ The bench press has also been one of the most frequently performed strengthtraining exercises and is a recommended testing mechanism by the American College of Sports Medicine for determining upperbody strength. ${ }^{36}$ All protocols included exercises that targeted major muscle groups for the development of strength and hypertrophy, although the variability in the number and type of exercises selected was so varied that determining the most effective exercise grouping cannot be made from these studies. Further research should include a comparison of SR to the TB protocols including the same exercises, with a number of exercises closer to that of Schoenfeld et al, ${ }^{6}$ which utilized 21 distinct exercises. The use of a great number of exercises would ensure that the muscles would be activated for the greatest potentiation of muscular strength development and hypertrophy. Further research should also investigate how a lower-frequency TB protocol may be compared with a higher-frequency SR protocol in college athletes, where time constraints due to schedule and college time contact rules may affect training.

The limited number of studies that evaluate trained male subjects with regard to high- or low-frequency training sessions, as well as the scarcity of literature available on this topic, makes it challenging to properly determine if a lower frequency of training sessions with an increase in volume can be successfully administered to other well-trained groups, such as those in college or professional athletics. Studies that incorporate frequency and volume modifications, as well as training protocols that include TB training sessions, can further justify or support the evidence presented in this CAT. As mentioned previously, future research should consider administering studies that focus on the well-trained athlete, particularly those who participate in sports that require increased muscle size and muscular strength development. This CAT should be reviewed in 2 years to determine whether additional best research evidence has been published that could aid in answering the focused clinical question. 


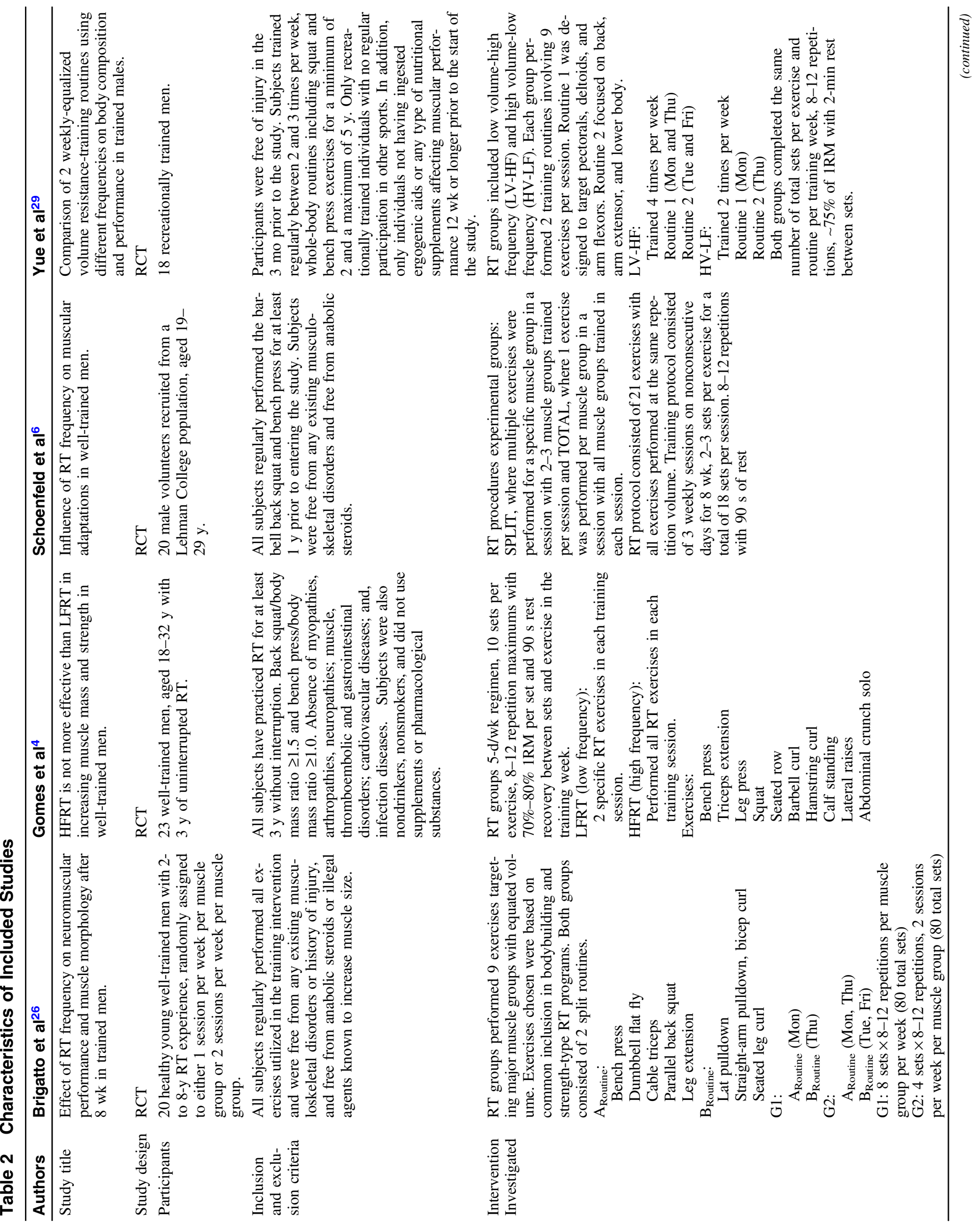




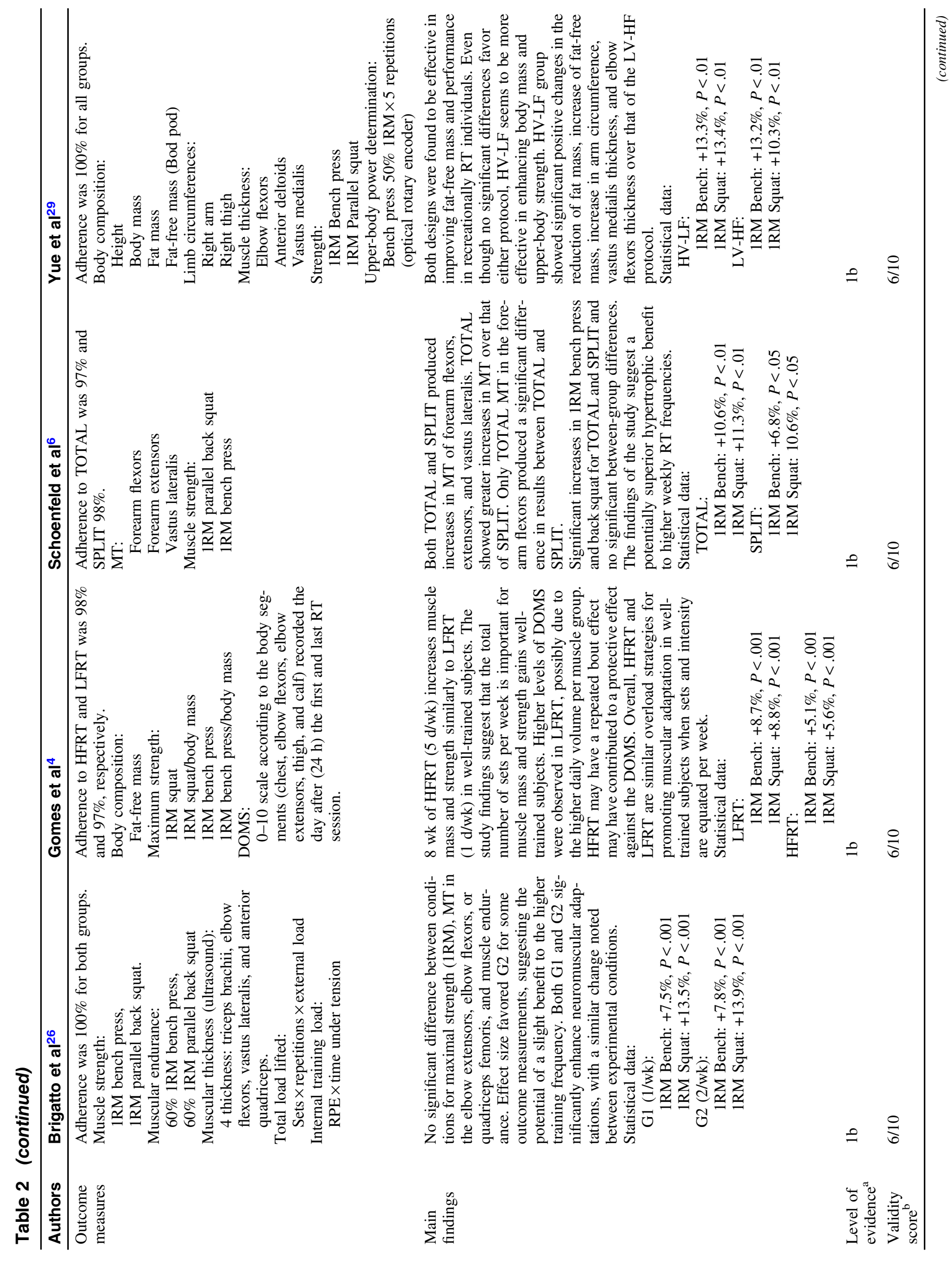




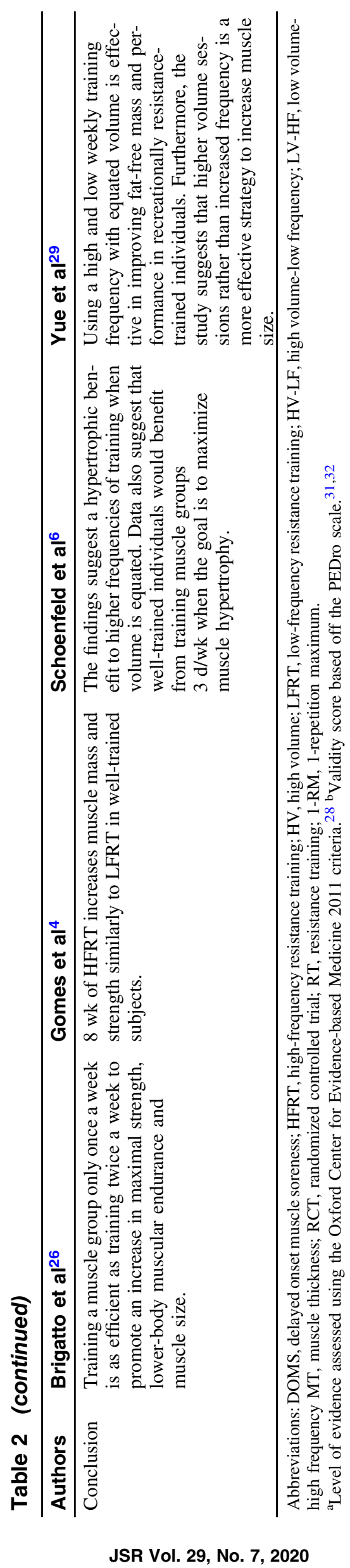




\section{References}

1. Candow DG, Burke DG. Effect of short-term equal-volume resistance training with different workout frequency on muscle mass and strength in untrained men and women. J Strength Cond Res. 2007; 21(1):204-207. PubMed ID: 17313289 doi:10.1519/00124278200702000-00037

2. Dankel SJ, Mattocks KT, Jessee MB, et al. Frequency: the overlooked resistance training variable for inducing muscle hypertrophy? Sports Med. 2017;47(5):799-805. PubMed ID: 27752983 doi:10.1007/ s40279-016-0640-8

3. Gentil P, Fischer B, Martorelli AS, Lima RM, Bottaro M. Effects of equal-volume resistance training performed one or two times a week in upper body muscle size and strength of untrained young men. J Sports Med Phys Fitness. 2015;55(3):144-149. PubMed ID: 24732784

4. Gomes GK, Franco CM, Nunes PRP, Orsatti FL. High-frequency resistance training is not more effective than low-frequency resistance training in increasing muscle mass and strength in well-trained men. J Strength Cond Res. 2019;33(7)(Suppl):S130-S139. doi:10.1519/ JSC.0000000000002559

5. Hoffman JR, Kraemer WJ, Fry AC, Deschenes M, Kemp M. The effects of self-selection for frequency of training in a winter conditioning program for football. J Strength Cond Res. 1990;4(3):76-82.

6. Schoenfeld BJ, Ratamess NA, Peterson MD, Contreras B, Tiryaki-Sonmez G. Influence of resistance training frequency on muscular adaptations in well-trained men. J Strength Cond Res. 2015;29(7):1821-1829. PubMed ID: 25932981 doi:10.1519/JSC. 0000000000000970

7. Simao R, de Tarso Veras Farinatti P, Polito MD, Maior AS, Fleck SJ. Influence of exercise order on the number of repetitions performed and perceived exertion during resistance exercises. $J$ Strength Cond Res. 2005;19(1):152-156. PubMed ID: 15705026

8. Weakley JJS, Till K, Read DB, et al. The effects of traditional, superset, and tri-set resistance training structures on perceived intensity and physiological responses. Eur J Appl Physiol. 2017;117: 1877-1889. PubMed ID: 28698987 doi:10.1007/s00421-017-3680-3

9. Campos GER, Luecke TJ, Wendeln HK, et al. Muscular adaptations in response to three different resistance-training regimens: specificity of repetition maximum training zones. Eur J Appl Physiol. 2002; 88(1-2):50-60. PubMed ID: 12436270 doi:10.1007/s00421-0020681-6

10. Colquhoun RJ, Gai CM, Aguilar D, et al. Training volume, not frequency, indicative of maximal strength adaptations to resistance training. J Strength Cond Res. 2018;32(5):1207-1213. PubMed ID: 29324578 doi:10.1519/JSC.0000000000002414

11. Potteiger JA, Judge LW, Cerny JA, Potteiger VM. Effects of altering training volume and intensity on body mass, performance, and hormonal concentrations in weight-event athletes. J Strength Cond Res. 1995;9(1):55-58.

12. Schoenfled BJ, Contreras B, Krieger J, et al. Resistance training volume enhances muscle hypertrophy but not strength in trained men. Med Sci Sports Exerc. 2018;51(1):94-103. doi:10.1249/MSS. 0000000000001764

13. Tsoukos A, Veligekas P, Brown LE, Terzis G, Bogdanis GC. Delayed effects of a low-volume, power-type resistance exercise session on explosive performance. J Strength Cond Res. 2017;32(3):643-650. doi:10.1519/JSC.0000000000001812

14. Wilson GJ, Newton RU, Murphy AJ, Humphries BJ. The optimal training load for the development of dynamic athletic performance. Med Sci Sports Exerc. 1993;25(11):1279-1286. PubMed ID: 8289617 doi:10.1249/00005768-199311000-00013
15. Ahtiainen JP, Pakarinen A, Markku A, Kraemer WJ, Hakkinen K. Short versus long rest period between the sets in hypertrophic resistance training: influence on muscle strength, size, and hormonal adaptations in trained men. J Strength Cond Res. 2005;19(3):572582. PubMed ID: 16095405

16. Buresh R, Berg K, French J. The effect of resistive exercise rest interval on hormonal response, strength, and hypertrophy with training. J Strength Cond Res. 2009;23(1):62-71. PubMed ID: 19077743 doi:10.1519/JSC.0b013e318185f14a

17. Willardson JM, Burkett LN. The effect of different rest intervals between sets on volume components and strength gains. J Strength Cond Res. 2008;22(1):146-152. PubMed ID: 18296968 doi:10.1519/ JSC.0b013e31815f912d

18. Hatfield DL, Kraemer WJ, Spiering BA, et al. The impact of velocity of movement on performance factors in resistance exercise. J Strength Cond Res. 2006;20(4):760-766. PubMed ID: 17194227

19. Brook MS, Wilkinson DJ, Mitchell WK, et al. Skeletal muscle hypertrophy adaptations predominate in the early stages of resistance exercise training, matching deuterium oxide-derived measures of muscle protein synthesis and mechanistic target of rapamycin complex1 signaling. FASEB J. 2015;29(11):4485-4496. PubMed ID: 26169934 doi:10.1096/fj.15-273755

20. Counts BR, Buckner SL, Mouser JG, et al. Muscle growth: to infinity and beyond? Muscle Nerve. 2017;56(6):1022-1030. PubMed ID: 28543604 doi: $10.1002 /$ mus. 25696

21. Hakkinen K, Newton RU, Gordon SC, et al. Changes in muscle morphology, electromyographic activity, and force production characteristics during progressive strength training in young and older men. J Gerontol. 1998;53(6):B415-B423. doi:10.1093/gerona/53A. 6.B415

22. Ratamess NA, Alvar BA, Evetock TK, et al. American College of Sports Medicine position stand: progression models in resistance training for healthy adults. Med Sci Sports Exerc. 2009;41(3):687708. doi:10.1249/MSS.0b013e3181915670

23. Damas F, Phillips S, Vechin FC, Ugrinowitsch C. A review of resistance training-induced changes in skeletal muscle protein synthesis and their contribution to hypertrophy. Sports Med. 2015;45(6):801-807. PubMed ID: 25739559 doi:10.1007/s40279015-0320-0

24. Schoenfeld BJ, Ogborn D, Krieger JW. Effects of resistance training frequency on measures of muscle hypertrophy: a systematic review and meta-analysis. Sports Med. 2016;46(11):1689-1697. PubMed ID: 27102172 doi:10.1007/s40279-016-0543-8

25. Baechle TR, Earle RW, eds. Essentials of Strength Training and Conditioning. 4th ed. Champaign, IL: Human Kinetics; 2008.

26. Brigatto FA, Braz TV, Zanini TCDC, et al. Effect of resistance training frequency on neuromuscular performance and muscle morphology after eight weeks in trained men. J Strength Cond Res. 2019;33(8):2104-2116. PubMed ID: 29528962 doi:10.1519/JSC. 0000000000002563

27. Rhea MR, Ball SD, Phillips WT, Burkett LN. A comparison of linear and daily undulating periodized programs with equated volume and intensity for strength. J Strength Cond Res. 2002;16(2):250-255. PubMed ID: 11991778

28. OCEBM Levels of Evidence Working Group*. The Oxford Levels of Evidence 2. Oxford Centre for Evidence-Based Medicine. https:// www.cebm.net/index.aspx?o=5653

29. Yue FL, Karsten B, Larumbe-Zabala E, Seijo M, Naclerio F. Comparison of 2 weekly-equalized volume resistance-training routines using different frequencies on body composition and performance in trained males. Appl Physiol Nutr Metab. 2018;43:475-481. 
30. Heaselgrave SR, Blacker J, Smeuninx B, McKendry J, Breen L. Dose-response relationship of weekly resistance-training volume and frequency on muscular adaptations in trained men. Int J Sports Physiol Perform. 2019;14(3):360-368. PubMed ID: 30160627 doi:10.1123/ijspp.2018-0427

31. De Morton NA. The PEDro scale is a valid measure of the methodological quality of clinical trials: a demographic study. Aust J Physiother. 2009;55(2):129-133. PubMed ID: 19463084 doi:10.1016/ S0004-9514(09)70043-1

32. Maher CG, Sherrington C, Herbert RD, Moseley AM, Elkins M. Reliability of the PEDro scale for rating quality of randomized controlled trials. Phys Ther. 2003;83(8):713-721. PubMed ID: 12882612 doi:10.1093/ptj/83.8.713
33. Kenn J. The Coach's Strength Training Playbook: Featuring the Tier System. Monterey, CA: Coaches Choice; 2003.

34. Rhea MR, Alvar BA, Burkett LN, Ball SD. A meta-analysis to determine the dose response for strength development. Med Sci Sports Exerc. 2003;35(3):456-464. PubMed ID: 12618576 doi:10. 1249/01.MSS.0000053727.63505.D4

35. Jakovljeic S, Karalejic M, Pajic Z, Jankovic N, Erculj F. Relationship between 1RM squat test results and explosive movements in professional basketball players. Acta Universitatis Carolinae: Kinanthropolgica. 2015;51(1):41-50.

36. Riebe D, Ehrman JK, Liguori G, Magal M, eds. ACSM's Guidelines for Exercise Testing and Prescription. 10th ed. Philadelphia, PA: Wolters Kluwer Health; 2018. 